\title{
Myocardial Edema Imaging in Acute Coronary Syndromes
}

\author{
Michael C. Walls, M.D. ${ }^{1}$, David Verhaert, M.D. ${ }^{2}$, and Subha V. Raman, M.D., M.S.E.E. ${ }^{1}$ \\ ${ }^{1}$ The Ohio State University, 473 W. $12^{\text {th }}$ Ave, Suite 200, Columbus, OH 43210, USA \\ ²Ziekenhuis Oost-Limburg, Schiepse Bos 6, 3600 Genk, Belgium
}

\section{Abstract}

Acute coronary syndromes (ACS) continue to be the most common morbid condition of industrialized nations. The advent of and technical improvements in revascularization and medical therapy have led to a steady decline in mortality rates. However, many patients who suffer unstable angina or myocardial infarction require further testing and risk stratification to guide therapeutic selection and prognosis assignment. Myocardial edema imaging with cardiac magnetic resonance (CMR) affords the ability to define the amount of myocardium at risk, refine estimates of prognosis and provide guidance for therapies with excellent sensitivity compared to standard clinical markers. This review will discuss the rationale for edema imaging, how it is performed using CMR and its potential clinical applications.

Water, water, everywhere,

Nor any drop to drink.

Samuel Taylor Coleridge. "The Rime of the Ancient Mariner", Lyrical Ballads 1798.

Myocardial edema imaging has captured the imagination of magnetic resonance scientists and clinicians worldwide. Coleridge's classic poem describes a wayward crew who appears to die while surrounded by water, yet who are restored to life after an intervention.

Similarly, increased appreciation that myocardial edema signifies the presence of myocytes at risk of death suggests that its recognition should prompt appropriate interventions to save myocardium. Technical advances, preclinical studies and clinical trials using MRI for edema imaging have made it increasingly useful for both research as well as clinical care. While myocardial edema may occur in many cardiovascular conditions, this review will focus on its utility in imaging the patient with acute coronary syndrome (ACS), which encompasses myocardial infarction and unstable angina.

\section{Clinical motivation for edema imaging in ACS}

There are 1.57 million admissions per year in the U.S. for suspected ACS ${ }^{1}$. Of those, roughly 330,000 present with ST-segment elevation myocardial infarction (STEMI), with 'ST elevation' referring to a pathognomonic finding of ongoing myocardial injury by routine 12-lead electrocardiography (ECG). Once this classic ECG finding is detected, established management guidelines are swiftly implemented, making the ECG a critical and essential first test in any patient presenting with symptoms or signs concerning for ACS. Management includes rapid delivery of well-defined medical therapies and transport to a cardiac

Address for correspondence: Subha V. Raman, MD, MSEE, Associate Professor, The Ohio State University, Columbus, OH 43210 , phone 614 293-8963, fax 614 293-5614, raman.1@ osu.edu.

Disclosures

Dr. Raman receives research support from Siemens 
catheterization laboratory capable of performing emergent percutaneous coronary intervention (PCI). The remaining 1.24 million Americans include those with unstable angina (UA) and non-ST-segment elevation myocardial infarction (NSTEMI), collectively termed non-ST elevation acute coronary syndromes (NSTE-ACS). Unlike patients presenting with STEMI whose diagnosis is usually obvious from history and ECG, patients presenting with NSTE-ACS often have delays in diagnosis due to atypical symptoms and equivocal electrocardiographic findings. Nonetheless, there are established diagnostic criteria and management pathways for patients with NSTE-ACS that pay particular attention to serological biomarkers of myocardial injury, most commonly the troponin assay ${ }^{2}$.

Thus far, the reader may note that edema imaging has not been mentioned. In the context of well-defined clinical, serological and electrocardiographic parameters in the evaluation of patients with ACS, imaging may have no added value unless it offers 1) greater accuracy in making the initial diagnosis, 2) lower risk with evaluation and management, and 3) refined risk stratification to the extent that management decision-making is influenced by imaging findings. This review will summarize why edema imaging with cardiac magnetic resonance (CMR) has the potential to address these three areas beyond techniques that are currently available. We will next review pathophysiological considerations that make myocardial edema an important target for imaging in ACS. The following sections offer evidence supporting magnetic resonance-based techniques as suitable noninvasive markers of myocardial edema and detail recent refinements in T2-based measurement techniques. Finally, we summarize current evidence supporting CMR-based edema imaging in various clinical settings and highlight areas where further investigation is needed.

\section{Myocardial edema in ACS: pathophysiology of the area at risk (AAR)}

The processes in ischemic myocardial cell injury and death have been well characterized ${ }^{3,4}$. Typical ACS result from rupture or erosion of an atherosclerotic plaque in an epicardial coronary artery (Figure 1). ACS may also result from vasospasm or occasionally from primary thrombotic events in the absence of significant epicardial atherosclerosis, though these mechanisms will not be considered in more detail in this review. Typically, the culprit coronary plaque leading to ACS is lipid-rich with a thin fibrous cap whose disruption exposes blood elements to intraplaque material and quickly cascades to superimposed thrombus formation that produces partial vs. complete, and intermittent vs. sustained coronary occlusion. As the vessel recanalizes, either spontaneously or through mechanical revascularization via angioplasty and stent placement, plaque debris and thrombotic material may embolize downstream and occlude the myocardial microcirculation producing so-called 'microvascular obstruction'.

The pathways from coronary occlusion and reperfusion to myocardial injury are numerous, with reduced myocardial blood flow relative to oxygen demand occurring alongside many other biochemical processes. Impaired delivery of oxygen to the myocardium causes the cessation of mitochondrial oxidative phosphorylation resulting in a depletion of adenosine triphosphate (ATP). A cellular shift to anaerobic glycolysis tries to maintain a steady source of ATP; however this process leads to accumulation of hydrogen ions and lactate, resulting in intracellular acidosis and the inhibition of glycolysis. It is this process that results in cellular membrane instability leading to alterations in ion concentrations due to a lack of ATP that is required for maintaining cellular ion homeostasis. Influx of sodium ions due to ischemia-activation of the sodium-hydrogen exchange leads to intracellular water increase ${ }^{5}$.

If ischemia persists then myocardial injury becomes irreversible with the necrosis extending from the endocardium to the subepicardium as classically described by Reimer and Jennings 
who termed the pathologic process the "wavefront phenomenon". ${ }^{6,7}$ However, reperfusion after the acute event further extends edema that gradually resolves as the injury heals. ${ }^{8}$

The amount of inadequately perfused myocardium distal to the coronary occlusion is defined as the area at risk (AAR). With sustained ischemia, the myocardium denoted by the AAR may go on to suffer irreversible injury. Being able to identify the AAR is a primary goal of edema imaging with CMR. Other modalities that have demonstrated feasibility in delineating AAR include single photon emission computed tomography (SPECT) ${ }^{9}$ and contrast echocardiography ${ }^{10}$, though neither have gained significant clinical use for such. This is likely due to a combination of limited sensitivity as well as limited therapeutic options directly targeting AAR beyond standard anatomy-driven reperfusion techniques. Increased sensitivity, lack of need for ionizing radiation or contrast agent and mechanistic underpinnings to AAR's development make CMR-based edema imaging an appealing alternative to either modality.

\section{Proton T2 relaxation time and myocardial edema}

The notion of detecting myocardial edema due to ischemic injury with magnetic resonance originated from animal models of acute $\mathrm{MI}$ and the earliest spin echo nuclear magnetic resonance techniques. ${ }^{11-13}$ Higgins et al. performed T2W spin echo acquisition of excised hearts twenty-four hours after inducing acute MI in 8 dogs. This demonstrated elevated T2 values in the regions of infarct that were linearly correlated with the percent water content of infarcted myocardium. More recently, Aletras et al. performed a study in 17 canines subjected to 90 minutes of coronary artery occlusion; these animals underwent $\mathrm{T} 2 \mathrm{~W}$ imaging with quantification of myocardial deformation using displacement encoding (DENSE) 2 days later. ${ }^{14}$ Volume of myocardium showing increased signal on T2W CMR correlated exactly with AAR defined by fluorescent microspheres injected at the time of coronary occlusion. Thus, this landmark study established T2W CMR as an accurate, noninvasive surrogate for AAR. This study also provided insight into the time course of edema resolution post-STEMI: eight dogs followed for an additional 2 months showed normalization of signal intensity on $\mathrm{T} 2 \mathrm{~W}$ imaging, with corresponding improvement in regional deformation.

\section{Techniques to quantify myocardial edema}

$\mathrm{T} 2$ is the exponential time constant which governs the rate of decay of transverse magnetization following the application of a radio frequency pulse. Tissue interactions between neighboring spins cause a loss of phase coherence, which results in a loss of transverse magnetization and reduced MR signal. With a spin echo acquisition, T2W image contrast is achieved by imaging with a long repetition time (TR) compared to tissue T1 (this reduces the $\mathrm{T} 1$ contribution to image contrast) and a long echo time (TE). The tissues with a longer T2 will have a higher signal in a T2W image, such as myocardium with increased water content (Figure 2). Importantly, the extent of protein binding also greatly influences $\mathrm{T} 2$ signal ${ }^{15}$. Other factors that influence $\mathrm{T} 2$ include changes in blood volume, hemoglobin oxygenation and mobile hydrogen on fatty acids ${ }^{16}$. Using a TE of 60-64 msec (the typical values used in clinical T2 CMR), the contribution of these factors and their impact on tissue with very long or very short T2 is minimal, which leaves free water as the main contributor of the T2 signal ${ }^{16}$.

Spin-echo CMR techniques have been used extensively to image myocardial edema ${ }^{11,17-21}$. As technology improved, more advanced spin echo techniques, such as turbo spin-echo and fast spin-echo have been used to acquire high resolution, black blood images of the heart with a single breathhold or single heartbeat. ${ }^{22,23}$ To enhance the sensitivity to tissue fluid while suppressing the fat signal, a third inversion pulse with a short TI technique termed 
short tau inversion recovery (STIR) was developed. The STIR pulse effectively suppressed the signal from short T1 tissues, highlighting the signal from long T1 and long T2 species, such as fluid and edematous tissue. ${ }^{22}$

Recent papers have acknowledged a set of challenges that limit the clinical acceptance of T2W-STIR for myocardial edema imaging. ${ }^{24-26}$ These limitations include phased-array coils causing progressive loss of signal in the posterior and lateral myocardial segments. Inadequate blood suppression that results from stagnant flow at the endocardial border may cause high signal from the blood pool that contaminates the myocardium. Rapid cardiac or respiratory motion impairing image quality. Finally, minor changes in sequence parameters such as TE and slice thickness in the setting of varying heart rate, for instance, considerably affect the qualitative appearance of T2W STIR images, affecting both reproducibility and accuracy.

To overcome these limitations, Giri et al. proposed a new quantitative T2 mapping sequence. ${ }^{27}$ Briefly, the $\mathrm{T} 2$ maps were generated by acquiring three $\mathrm{T} 2 \mathrm{~W}$ images, each with a different T2 preparation time $(0 \mathrm{~ms}, 24 \mathrm{~ms}$ and $55 \mathrm{~ms}$ respectively; TR $=3 \times \mathrm{R}-\mathrm{R}$, total acquisition time of 7 heartbeats). To correct for motion between images, a fast variational non-rigid registration algorithm was used, aligning all T2-prepared frames to the center frame. ${ }^{28}$ Each of the three images produced a signal intensity that corresponded to a function of T2-decay that was fitted using a linear 2-parameter model after logarithmic transformation to derive the T2-value of each pixel. This practical technique allowed the investigators to quantitate the absolute $\mathrm{T} 2$ value of myocardium in porcine and human subjects while overcoming the limitations of myocardial motion, surface coil intensity variation, high subendocardial signal from static blood, and the subjective nature of image interpretation.

Our group recently tested this technique in a cohort of 27 ACS patients with either STEMI or NSTEMI ${ }^{29}$. Significant differences could be detected in myocardial regions of acute infarct versus remote segments ( $69 \pm 6 \mathrm{~ms}$ versus $56 \pm 3 \mathrm{~ms}$ ). Furthermore, this work showed $\mathrm{T} 2$ values of $59 \pm 6 \mathrm{~ms}$ in regions with microvascular obstruction (MO), consistent with the expected $\mathrm{T} 2$ shortening in regions of MO. T2 mapping consistently provided adequate image data for T2 measurement in a population in whom 30\% STIR yielded inadequate $\mathrm{T} 2 \mathrm{~W}$ images, with many patients unable to breathhold and others with irregular heart rhythms.

Some have suggested that since LGE shows hyperenhancement in regions of interstitial edema as well as irreversible necrosis shortly post-MI, that LGE may be sufficient to delineate $\mathrm{AAR}^{30}$. However, this has not been our experience particularly when using $\mathrm{T} 2$ mapping that consistently shows regions of increased $\mathrm{T} 2$ in myocardium at risk not subtended by hyperenhancement on LGE, even when using modest (e.g. 2SD above the mean of normal myocardium) thresholds.

\section{Clinical applications}

Human studies have demonstrated clinical utility of detecting increased myocardial T2 signal in patients with known or suspected acute coronary syndromes. Abdel-Aty et al. performed $\mathrm{T} 2 \mathrm{~W}$ and late gadolinium enhanced (LGE) imaging in patients with either acute or chronic MI. ${ }^{31}$ Patients with acute MI within two weeks of presentation demonstrated increased myocardial signal intensity on $\mathrm{T} 2 \mathrm{~W}$ myocardial imaging, whereas patients with chronic infarcts showed no increased signal on T2W imaging. LGE patterns were not significantly different between patients with acute vs. chronic MI. These findings underscore the potential value of $\mathrm{T} 2 \mathrm{~W}$ edema imaging in the patient with known coronary disease who presents with signs and symptoms concerning for but not definitive of ACS. 
Cury et al. demonstrated the incremental value of T2W-CMR in patients presenting to the emergency department ${ }^{32}$. Detection of ACS upon initial evaluation with comprehensive CMR examination that included T2W imaging was compared to presence/absence of ACS by clinical criteria at discharge from the hospital; overall diagnostic accuracy increased to $93 \%$ vs. $84 \%$ if using only on cine, rest perfusion and LGE findings.

In patients who suffer from acute STEMI, several groups have shown that after coronary angiography (typically with percutaneous intervention i.e. angioplasty and stent placement), T2-CMR delineates AAR ${ }^{29,33,34}$. Berry et al., using a T2-prepared SSFP sequence, further showed superior performance of AAR quantification compared to electrocardiographic and angiographic estimates. These studies offer T2-CMR as a useful surrogate endpoint in trials of novel therapeutics to reduce AAR post-STEMI. What remains to be determined from these types of studies is how clinical management should be modified based on delineation of AAR post-revascularization. This likely requires parallel development of better therapeutics beyond mechanical reperfusion and current adjuvant drug therapies. Knowing the location and extent of edema, it is intriguing to consider how edema imaging may have the power to predict life-threatening complications post-STEMI. These complications include cardiogenic shock, papillary muscle rupture leading to acute mitral regurgitation (Figure 3), left ventricular pseudoaneurysm, ventricular septal defect, and ventricular arrhythmias, which are the leading causes of death in acute MI patients.

With availability of serum biomarkers that are highly sensitive to myocardial injury, identifying edema by $\mathrm{T} 2 \mathrm{CMR}$ would be particularly useful if myocardium at risk could be detected even before serum biomarker elevation (Figure 4). This was demonstrated by Abdel-Aty et al. in a meticulous study of eleven dogs eliciting ischemia and reperfusion injury ${ }^{35}$. Increased myocardial signal intensity on T2W imaging occurred at $28 \pm 4$ min after experimental coronary occlusion, a time point at which serum troponin level was still normal, suggesting increased sensitivity to early myocardial changes with $\mathrm{T} 2$ edema detection vs. a widely-used serum biomarker of myocardial injury.

Applying this concept to patients, it has been shown that 53\% of individuals presenting with NSTE-ACS whose initial cardiac biomarkers were negative using a high-sensitivity troponin-I assay have detectable myocardial edema by $\mathrm{T} 2 \mathrm{~W}-\mathrm{CMR}^{36}$. This suggests that patients without declarative changes by initial biomarkers or other routine testing such as electrocardiography may be missed by conventional clinical assessment, and T2-CMR improves early identification of individuals with myocardium at risk without the need for stress testing. While many patients in this study went on to require revascularization in the form of either percutaneous stent placement or bypass surgery, presence of edema as detected by T2W-CMR predicted major adverse cardiovascular events at 6 months postACS regardless of whether or not mechanical revascularization was performed, underscoring the notion that myocardial edema is a significant adverse prognostic finding in ACS.

Further studies are needed to demonstrate whether or not individuals with NSTE-ACS identified to have myocardium at risk by T2-CMR benefit from earlier treatment with aggressive medical therapies such as parenteral anti-platelet agents. Also uncertain at this time is whether or not such patients warrant more rapid invasive angiography, similar to patients with STEMI who have readily-identifiable myocardium at risk by electrocardiography. For these patients, the adage 'time is muscle' has driven remarkable advances in pre-hospital management, emergency department triage and interventional care. Recognition of myocardium at risk through T2-CMR may translate to similar advances in managing patients with NSTE-ACS, who notably comprise $70-80 \%$ of all ACS. Similarly, further study is needed to test the hypothesis that ACS patients without myocardium at risk 
do well with conservative management without the risks of invasive angiography and potent anticoagulant/anti-platelet regimens.

In current practice, incorporation of such an examination in the acute setting of ACS would be limited by the typical availability of CMR which may not even be comparable to that of SPECT examination in most hospitals. A potential solution would be to consider CMR as a modality that requires appropriate physician expertise, technical staff and resource allocation similar to SPECT for patients presenting off-hours with suspected ACS. Once available under such circumstances, randomized clinical trials can be done to test the hypothesis that identification of myocardium at risk by T2-CMR helps stratify patients with ACS and guide timing of invasive evaluation and intensity of adjuvant therapies (Figure 5).

\section{Conclusions}

The interrupted delivery of oxygen-rich blood to the myocardium produces an ischemic cascade that, if left untreated, can lead to irreversible injury. CMR with T2 imaging can detect edema prior to irreversible injury and can also delineate area at risk beyond any irreversibly-injured myocardium. In the appropriately selected patient, CMR may deliver earlier and more accurate risk stratification, prognostication, and refined treatment selection for ACS. Recognizing that T2-CMR accurately delineates myocardium at risk, translational research that uses edema imaging can guide development of novel therapeutics that restore life to at-risk myocytes. While such therapies undergo development, improved CMR-based myocardial edema imaging techniques available today that perform robustly in typical patients with ischemic heart disease can deliver low-risk imaging to improve diagnosis, refine management and improve outcomes for patients with acute coronary syndromes.

\section{Acknowledgments}

\section{Sources of Funding}

Dr. Raman is supported in part by NIH R01 HL095563 and HL102450

\section{REFERENCES}

1. Roger VL, Go AS, Lloyd-Jones DM, et al. Heart Disease and Stroke Statistics--2011 Update: A Report from the American Heart Association. Circulation. 2010; 123(4):e18-e209. [PubMed: 21160056]

2. Anderson JL, Adams CD, Antman EM, et al. ACC/AHA 2007 guidelines for the management of patients with unstable angina/non-ST-Elevation myocardial infarction: a report of the American College of Cardiology/American Heart Association Task Force on Practice Guidelines (Writing Committee to Revise the 2002 Guidelines for the Management of Patients With Unstable Angina/ Non-ST-Elevation Myocardial Infarction) developed in collaboration with the American College of Emergency Physicians, the Society for Cardiovascular Angiography and Interventions, and the Society of Thoracic Surgeons endorsed by the American Association of Cardiovascular and Pulmonary Rehabilitation and the Society for Academic Emergency Medicine. J Am Coll Cardiol. 2007; 50(7):e1-e157. [PubMed: 17692738]

3. Reimer KA, Ideker RE. Myocardial ischemia and infarction: anatomic and biochemical substrates for ischemic cell death and ventricular arrhythmias. Hum Pathol. 1987; 18(5):462-475. [PubMed: 2952574]

4. Buja L. Myocardial ischemia and reperfusion injury. Cardiovascular Pathology. 2005; 14(4):170175. [PubMed: 16009313]

5. Avkiran M. Basic biology and pharmacology of the cardiac sarcolemmal sodium/hydrogen exchanger. J Card Surg. 2003; 18 Suppl 1:3-12. [PubMed: 12691374] 
6. Reimer KA, Lowe JE, Rasmussen MM, Jennings RB. The wavefront phenomenon of ischemic cell death 1. Myocardial infarct size vs duration of coronary occlusion in dogs. Circulation. 1977; 56(5): 786-794. [PubMed: 912839]

7. Reimer KA, Jennings RB. The "wavefront phenomenon" of myocardial ischemic cell death. II. Transmural progression of necrosis within the framework of ischemic bed size (myocardium at risk) and collateral flow. Lab Invest. 1979; 40(6):633-644. [PubMed: 449273]

8. Wisenberg G, Prato FS, Carroll SE, Turner KL, Marshall T. Serial nuclear magnetic resonance imaging of acute myocardial infarction with and without reperfusion. Am Heart J. 1988; 115(3): 510-518. [PubMed: 3344656]

9. Sinusas AJ, Trautman KA, Bergin JD, et al. Quantification of area at risk during coronary occlusion and degree of myocardial salvage after reperfusion with technetium-99m methoxyisobutyl isonitrile. Circulation. 1990; 82(4):1424-1437. [PubMed: 2401074]

10. Iliceto S, Marangelli V, Marchese A, Amico A, Galiuto L, Rizzon P. Myocardial contrast echocardiography in acute myocardial infarction. Pathophysiological background and clinical applications. Eur Heart J. 1996; 17(3):344-353. [PubMed: 8737209]

11. Higgins CB, Herfkens R, Lipton MJ, et al. Nuclear magnetic resonance imaging of acute myocardial infarction in dogs: alterations in magnetic relaxation times. Am J Cardiol. 1983; 52(1): 184-188. [PubMed: 6858909]

12. Scholz TD, Martins JB, Skorton DJ. NMR relaxation times in acute myocardial infarction: relative influence of changes in tissue water and fat content. Magn Reson Med. 1992; 23(1):89-95. [PubMed: 1734185]

13. Karolle BL, Carlson RE, Aisen AM, Buda AJ. Transmural distribution of myocardial edema by NMR relaxometry following myocardial ischemia and reperfusion. Am Heart J. 1991; 122(3 Pt 1): 655-664. [PubMed: 1877442]

14. Aletras AH, Tilak GS, Natanzon A, et al. Retrospective determination of the area at risk for reperfused acute myocardial infarction with $\mathrm{T} 2$-weighted cardiac magnetic resonance imaging: histopathological and displacement encoding with stimulated echoes (DENSE) functional validations. Circulation. 2006; 113(15):1865-1870. [PubMed: 16606793]

15. Hazlewood CF, Chang DC, Nichols BL, Woessner DE. Nuclear magnetic resonance transverse relaxation times of water protons in skeletal muscle. Biophys J. 1974; 14(8):583-606. [PubMed: 4853385]

16. Bottomley PA, Foster TH, Argersinger RE, Pfeifer LM. A review of normal tissue hydrogen NMR relaxation times and relaxation mechanisms from 1-100 MHz: dependence on tissue type, NMR frequency, temperature, species, excision, and age. Med Phys. 1984; 11(4):425-448. [PubMed: 6482839]

17. Brown JJ, Peterson TM, Slutsky RA. Regional myocardial blood flow, edema formation, and magnetic relaxation times during acute myocardial ischemia in the canine. Invest Radiol. 1985 20(5):465-471. [PubMed: 4044191]

18. Dymarkowski S, Ni Y, Miao Y, et al. Value of t2-weighted magnetic resonance imaging early after myocardial infarction in dogs: comparison with bis-gadolinium-mesoporphyrin enhanced T1weighted magnetic resonance imaging and functional data from cine magnetic resonance imaging. Invest Radiol. 2002; 37(2):77-85. [PubMed: 11799331]

19. Choi SI, Jiang CZ, Lim KH, et al. Application of breath-hold T2-weighted, first-pass perfusion and gadolinium-enhanced T1-weighted MR imaging for assessment of myocardial viability in a pig model. J Magn Reson Imaging. 2000; 11(5):476-480. [PubMed: 10813856]

20. Bouchard A, Reeves RC, Cranney G, Bishop SP, Pohost GM. Assessment of myocardial infarct size by means of T2-weighted 1H nuclear magnetic resonance imaging. Am Heart J. 1989; 117(2): 281-289. [PubMed: 2916403]

21. Johnston DL, Homma S, Liu P, et al. Serial changes in nuclear magnetic resonance relaxation times after myocardial infarction in the rabbit: relationship to water content, severity of ischemia, and histopathology over a six-month period. Magn Reson Med. 1988; 8(4):363-379. [PubMed: 3231066]

22. Simonetti OP, Finn JP, White RD, Laub G, Henry DA. "Black blood" T2-weighted inversionrecovery MR imaging of the heart. Radiology. 1996; 199(1):49-57. [PubMed: 8633172] 
23. Stehling MK, Holzknecht NG, Laub G, Bohm D, von Smekal A, Reiser M. Single-shot T1- and T2-weighted magnetic resonance imaging of the heart with black blood: preliminary experience. MAGMA. 1996; 4(3-4):231-240. [PubMed: 9220412]

24. Abdel-Aty H, Simonetti O, Friedrich MG. T2-weighted cardiovascular magnetic resonance imaging. J Magn Reson Imaging. 2007; 26(3):452-459. [PubMed: 17729358]

25. Arai AE. Using magnetic resonance imaging to characterize recent myocardial injury: utility in acute coronary syndrome and other clinical scenarios. Circulation. 2008; 118(8):795-796. [PubMed: 18711021]

26. Pennell D. Myocardial salvage: retrospection, resolution, and radio waves. Circulation. 2006; 113(15):1821-1823. [PubMed: 16618830]

27. Giri S, Chung Y-C, Merchant A, et al. T2 quantification for improved detection of myocardial edema. Journal of Cardiovascular Magnetic Resonance. 2009; 11(1):56. [PubMed: 20042111]

28. Giri S, Xue H, Shah S, et al. Inline non-rigid motion-corrected t 2 mapping of myocardium. 13th Annual SCMR Scientific Sessions. Volume 12(Suppl I). Phoenix, AZ USA. Journal of Cardiovascular Magnetic Resonance. 2010:P229.

29. Verhaert DTP, Giri S, Mihai G, Rajagopalan S, Simonetti OP, Raman SV. Direct T2 quantification to detect myocardial edema in patients with acute ischemic injury. JACC Cardiovasc Imaging. 2011; 4(3):269-278. [PubMed: 21414575]

30. Wince WB, Kim RJ. Molecular imaging: T2-weighted CMR of the area at risk--a risky business? Nat Rev Cardiol. 2010; 7(10):547-549. [PubMed: 20865026]

31. Abdel-Aty H. Delayed Enhancement and T2-Weighted Cardiovascular Magnetic Resonance Imaging Differentiate Acute From Chronic Myocardial Infarction. Circulation. 2004; 109(20): 2411-2416. [PubMed: 15123531]

32. Cury RC, Shash K, Nagurney JT, et al. Cardiac magnetic resonance with T2-weighted imaging improves detection of patients with acute coronary syndrome in the emergency department. Circulation. 2008; 118(8):837-844. [PubMed: 18678772]

33. Berry C, Kellman P, Mancini C, et al. Magnetic resonance imaging delineates the ischemic area at risk and myocardial salvage in patients with acute myocardial infarction. Circ Cardiovasc Imaging. 2010; 3(5):527-535. [PubMed: 20631034]

34. Friedrich MG, Abdel-Aty H, Taylor A, Schulz-Menger J, Messroghli D, Dietz R. The salvaged area at risk in reperfused acute myocardial infarction as visualized by cardiovascular magnetic resonance. J Am Coll Cardiol. 2008; 51(16):1581-1587. [PubMed: 18420102]

35. Abdel-Aty H, Cocker M, Meek C, Tyberg JV, Friedrich MG. Edema as a very early marker for acute myocardial ischemia: a cardiovascular magnetic resonance study. J Am Coll Cardiol. 2009; 53(14):1194-1201. [PubMed: 19341860]

36. Raman SV, Simonetti OP, Winner MW 3rd, et al. Cardiac magnetic resonance with edema imaging identifies myocardium at risk and predicts worse outcome in patients with non-ST-segment elevation acute coronary syndrome. J Am Coll Cardiol. 2010; 55(22):2480-2488. [PubMed: 20510215] 

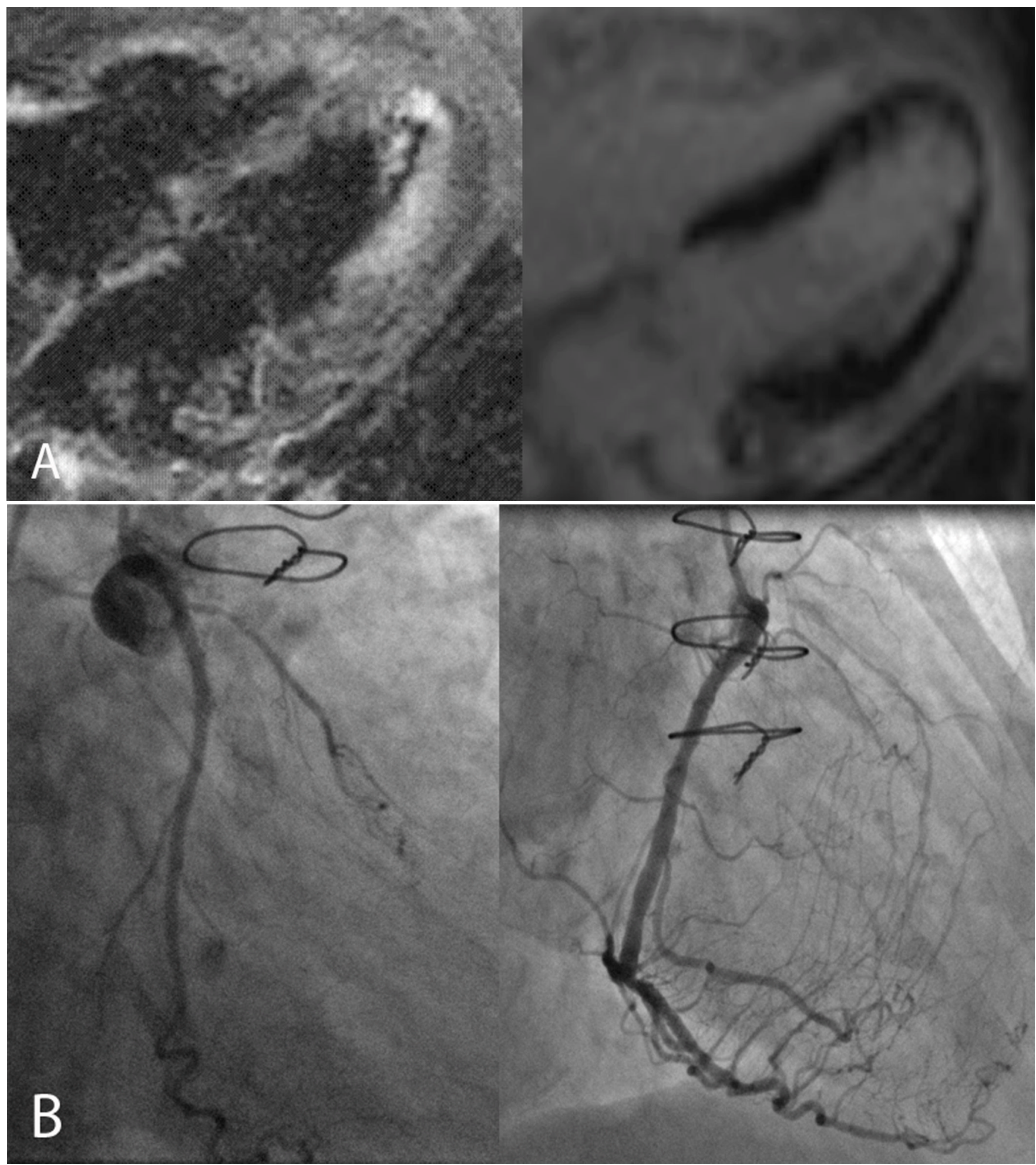

Figure 1.

A 74 year-old female with known history of an occluded saphenous vein graft to the first obtuse marginal artery presented to her cardiologist with recurrent angina. A. CMR examination included T2W-STIR imaging that revealed edema along the mid to apical 
anterior and anterolateral segments (arrow, left panel) with a reduced area of infarct scar in the same distribution (arrow, right panel) correlating with the area at risk. B. Subsequent invasive angiography identified ostial left anterior descending (LAD) coronary artery occlusion (left panel) with collateral vessels from the right coronary artery (right panel) supplying flow to the mid and distal LAD. This patient was ineligible for percutaneous or surgical revascularization secondary to her coronary anatomy and was successfully treated medically. 


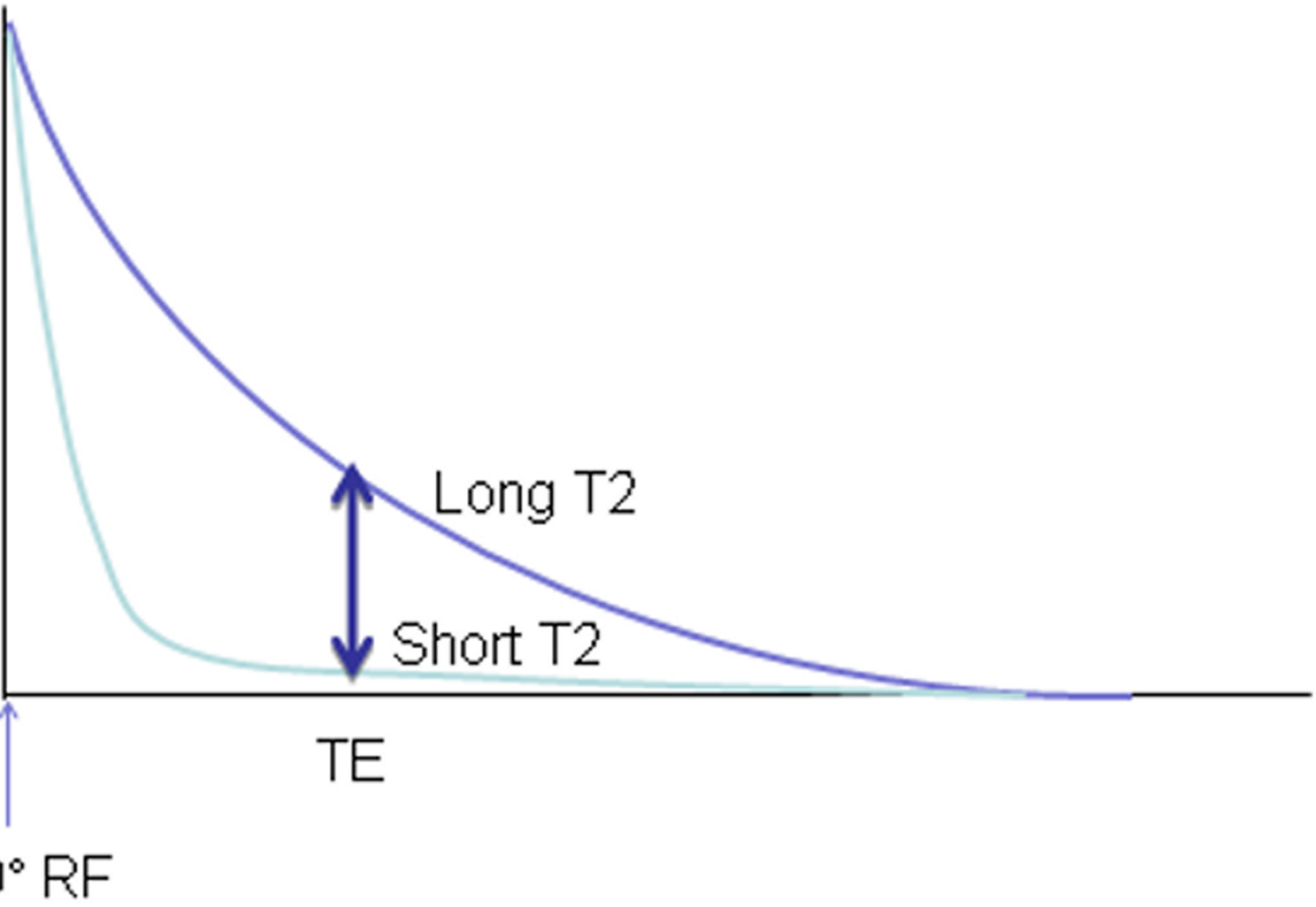

Figure 2.

The basic mechanism of T2-weighted magnetic resonance imaging involves highlighting contrast between spins with longer T2 (upper curve) and those with shorter T2 (lower curve). This is done with a simple spin-echo acquisition by collecting signal after a certain echo time (TE) following application of radiofrequency (RF) energy; T2-weighted sequences use longer TEs to maximize contrast between spins with long vs. short T2 values. 


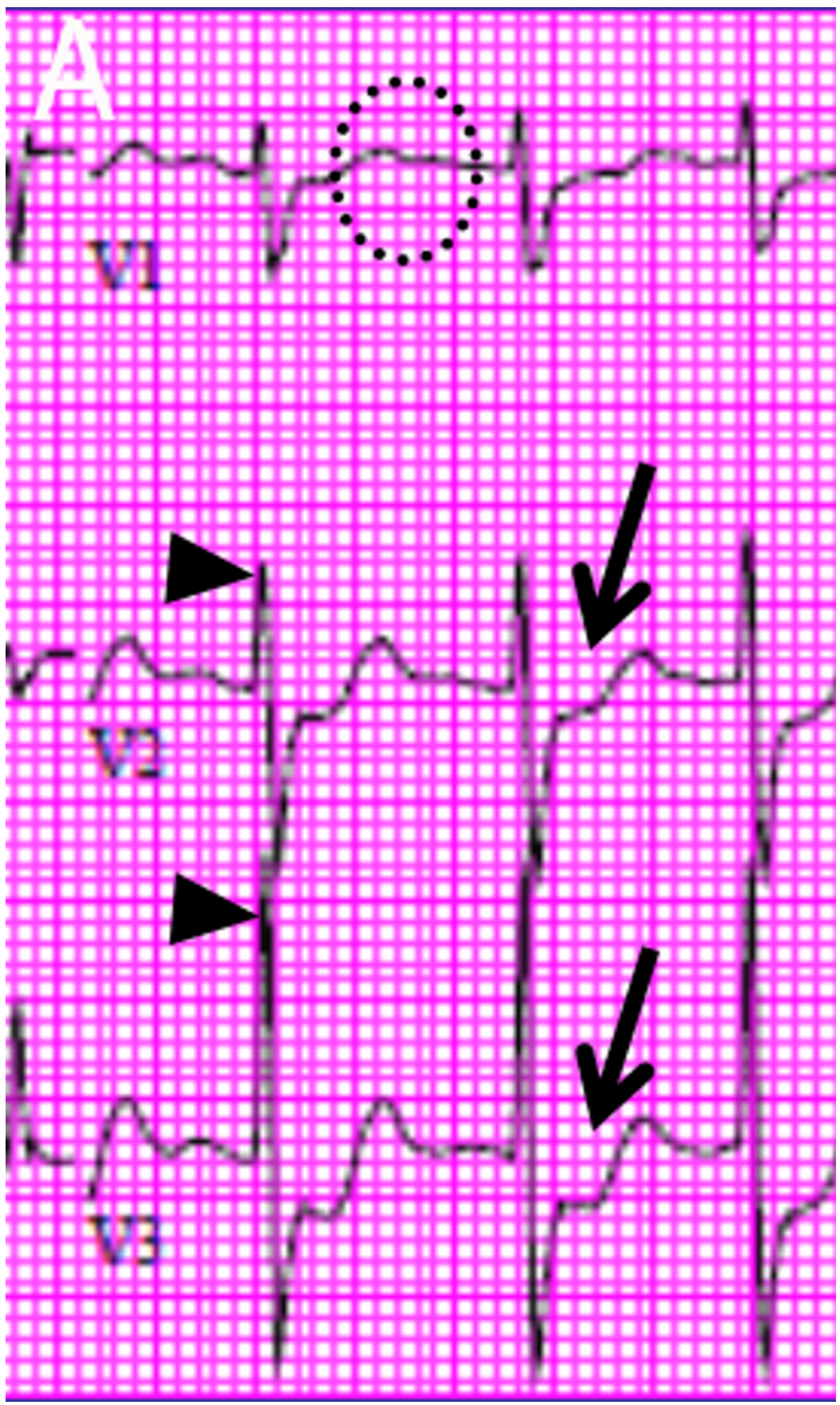




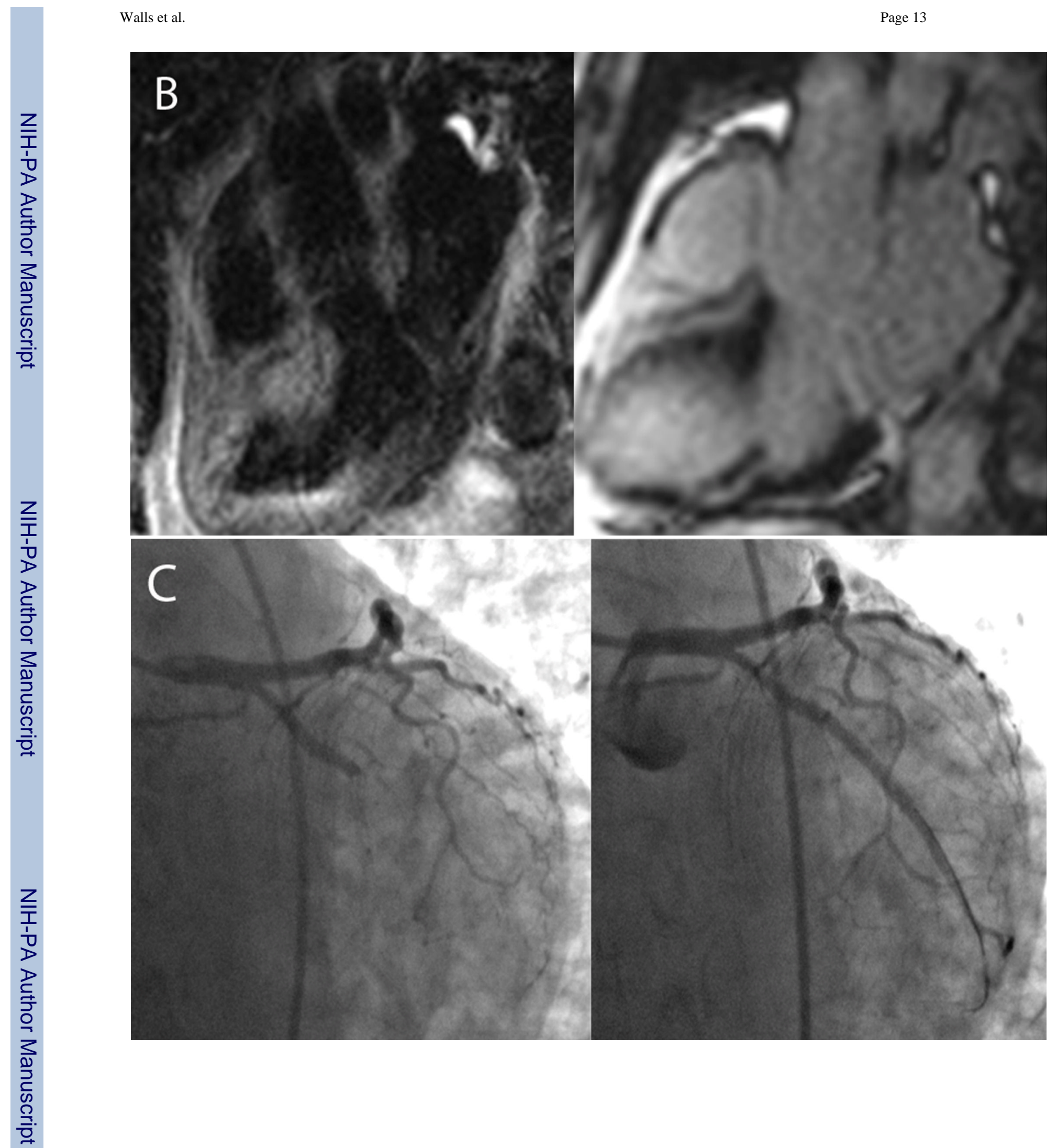

J Magn Reson Imaging. Author manuscript; available in PMC 2012 December 1. 


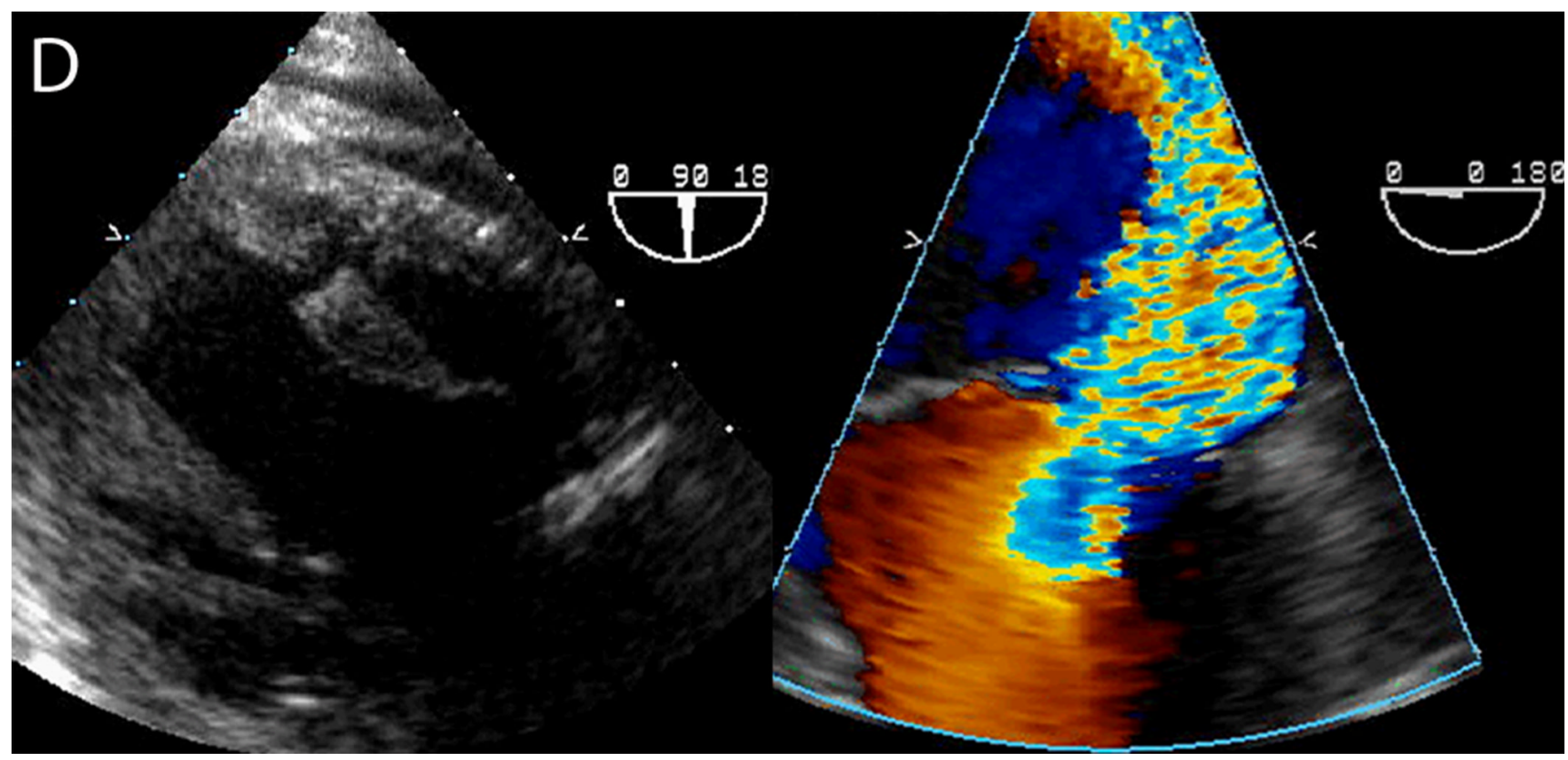

Figure 3.

An 83 year-old male presented to the emergency department with nausea, vomiting and abdominal pain. A. Electrocardiography showed posterior wall myocardial injury, indicated by ST depression (arrows), prominent $\mathrm{R}$ waves (arrowheads) and upright $\mathrm{T}$ waves (dotted circle) in leads V1 through V3. Due to coagulopathy and initial resolution of symptoms with medical therapies, invasive angiography was deferred. B. CMR examination performed within 1 hour of admission included T2W-STIR imaging that revealed edema along the inferolateral wall at the insertion of the posteromedial papillary muscle (left panel, dotted circle) without appreciable hyperenhancement on late post-gadolinium imaging (right panel). C. Subsequent angiography after resolution of coagulopathy showed a completed occluded circumflex coronary artery (left panel, circle), which was successfully recanalized via percutaneous coronary intervention (right panel). D. On day 3 after stent placement, the patient became suddenly diaphoretic with hypotension and pulmonary edema; new systolic murmur prompted transesophageal echocardiography that identified papillary muscle rupture that was promptly repaired. 

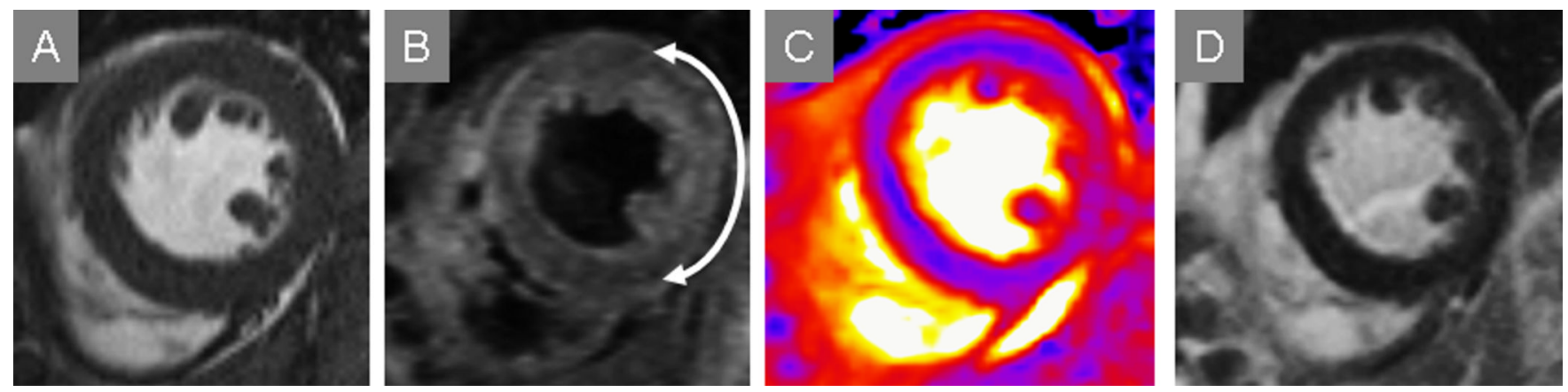

Figure 4.

T2-CMR affords identification of myocardium at risk even when irreversibly-injured myocardium is not apparent by either serum biomarkers such as troponin or other CMR markers such as late gadolinium enhancement. This patient's SSFP imaging suggested a lateral wall motion abnormality but edema was not apparent with this technique (A). Conventional T2-weighted STIR (B) and T2-mapping (C) both showed myocardial edema as increased T2 in the lateral wall (arrow delineates arc of involvement), while late postgadolinium imaging (D) showed no hyperenhancement consistent with lack of evident myocardial necrosis. 


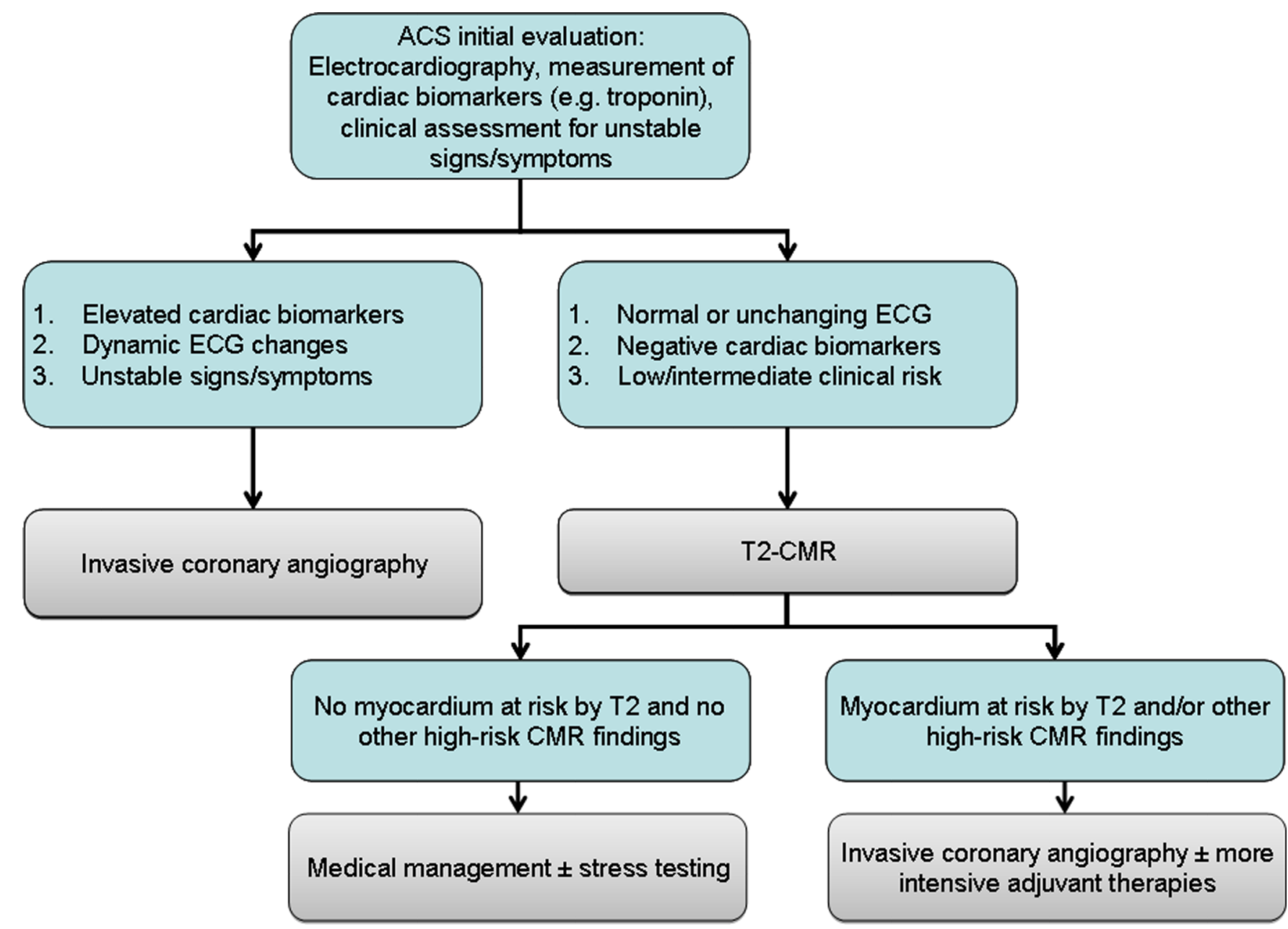

Figure 5.

A potential scheme for incorporation of T2-CMR in the evaluation of ACS patients is shown, with the caveat that randomized trials are needed to generate essential evidence that T2-CMR-guided management is comparatively effective compared to current strategies that emphasize invasive angiography in all but the lowest risk ACS patients. 